\title{
An Algorithm to Classify the Asymptotic Set Associated to a Polynomial Mapping
}

\author{
Nguyen Thi Bich Thuy \\ IBILCE, UNESP, Universidade Estadual Paulista, "Júlio de Mesquita", São José do Rio Preto, São Paulo, Brazil \\ Email: bichthuy@ibilce.unesp.br
}

How to cite this paper: Thuy, N.T.B. (2017) An Algorithm to Classify the Asymptotic Set Associated to a Polynomial Mapping. American Journal of Computational Mathematics, 7, 109-126.

https://doi.org/10.4236/ajcm.2017.71010

Received: January 18, 2017

Accepted: March 28, 2017

Published: March 31, 2017

Copyright $\odot 2017$ by author and Scientific Research Publishing Inc. This work is licensed under the Creative Commons Attribution International License (CC BY 4.0).

http://creativecommons.org/licenses/by/4.0/

\section{c) (†) Open Access}

\begin{abstract}
We provide an algorithm to classify the asymptotic sets of the dominant polynomial mappings $F: \mathbb{C}^{3} \rightarrow \mathbb{C}^{3}$ of degree 2 , using the definition of the so-called "façons" in [2]. We obtain a classification theorem for the asymptotic sets of dominant polynomial mappings $F: \mathbb{C}^{3} \rightarrow \mathbb{C}^{3}$ of degree 2 . This algorithm can be generalized for the dominant polynomial mappings $F: \mathbb{C}^{n} \rightarrow \mathbb{C}^{n}$ of degree $d$, with any $(n, d) \in\left(\mathbb{N}^{*}\right)^{2}$.
\end{abstract}

\section{Keywords}

Algorithms, Polynomial Mappings, Asymptotic Sets, Façons

\section{Introduction}

Let $F: \mathbb{C}^{n} \rightarrow \mathbb{C}^{n}$ be a polynomial mapping. Let us denote by $S_{F}$ the set of points at which $F$ is non proper, i.e.,

$S_{F}=\left\{a \in \mathbb{C}^{n}\right.$ such that $\exists\left\{\xi_{k}\right\}_{k \in \mathbb{N}} \subset \mathbb{C}^{n},\left|\xi_{k}\right|$ tends to infinity and $F\left(\xi_{k}\right)$ tends to $\left.a\right\}$, where $\left|\xi_{k}\right|$ is the Euclidean norm of $\xi_{k}$ in $\mathbb{C}^{n}$. The set $S_{F}$ is called the asymptotic set of $F$. The comprehension of the structure of this set is very important by its relation with the Jacobian Conjecture. In the 90 's, Jelonek studied this set in a deep way and described the principal properties. One of the important results is that, if $F$ is dominant, i.e., $F\left(\mathbb{C}^{n}\right)=\mathbb{C}^{n}$, then $S_{F}$ is an empty set or a hypersurface [1].

Notice that it is sufficient to define $S_{F}$ by considering sequences $\left\{\xi_{k}\right\}$ tending to infinity in the following sense: each coordinate of these sequences either tends to infinity or converges. In [2], the sequences tending to infinity such that their images tend to the points in $S_{F}$ are labeled in terms of "façons", as follows: We rank the coordinates of $\xi_{k}$ into three categories: 1) the coordinates tending to infinity (this cotegories is not empty); 2) the coordinates 
$\left(x_{k, i}\right)$ such that $\lim _{k \rightarrow \infty} x_{k, i}$ is a complex number "independant on the point $a$ in a neighborhood $a$ in $S_{F}$ ". This means that there exists the points neighbors of a in $S_{F}$ and the sequences $\left\{\xi_{k}^{\prime}\right\}$ such that $\lim _{k \rightarrow \infty} F\left(\xi_{k}^{\prime}\right)=a^{\prime}$ and $\left.\lim _{k \rightarrow \infty} x_{k, i}^{\prime}=\lim _{k \rightarrow \infty} x_{k, i}, 3\right)$ the coordinates $\left(x_{k, i}\right)$ such that $\lim _{k \rightarrow \infty} x_{k, i}$ is a complex number "dependant on the point $a$ ". This means that there not exist such points $a^{\prime}$ neighbors of $a$ in $S_{F}$. The example 2.5 illustrates these three categories.

We define a "façon" of the point $a \in S_{F}$ as a $(p, q)$-tuple $\left(i_{1}, \cdots, i_{p}\right)\left[j_{1}, \cdots, j_{q}\right]$ of integers where $x_{k, i_{r}}^{a}$ tends to infinity for $r=1, \cdots, p$ and, for $s=1, \cdots, q$, the sequence $x_{k, j_{s}}^{a}$ tends to a complex number independently on the point a when a describes locally $S_{F}$ (definition 2.7 ).

The aim of this paper is to provide an algorithm to classify the asymptotic sets of dominant polynomial mappings $F: \mathbb{C}^{3} \rightarrow \mathbb{C}^{3}$ of degree 2 , using the definition of "façons" in [2], and then generalize this algorithm for the general case. One important tool of the algorithm is the notion of pertinent variables. The idea of the notion of pertinent variables is the following: Let $F=\left(F_{1}, F_{2}, F_{3}\right): \mathbb{C}^{3} \rightarrow \mathbb{C}^{3}$ be a dominant polynomial mapping of degree 2 such that $S_{F} \neq \varnothing$. We fix a façon $\kappa$ of $F$ and assume that $\left\{\xi_{k}\right\}$ is a sequence tending to infinity with the façon $\kappa$ such that $F\left(\xi_{k}\right)$ tends to a point of $S_{F}$. Since the degree of $F$ is 2 then each coordinate $F_{1}, F_{2}$ and $F_{3}$ of $F$ is a linear combination of $f_{1}=x_{1}, f_{2}=x_{2}$, $f_{3}=x_{3}, f_{4}=x_{1} x_{2}, f_{5}=x_{2} x_{3}$ and $f_{6}=x_{3} x_{1}$. We call a pertinent variable of $F$ with respect to the façon $\kappa$ a minimum linear combination of $f_{1}, \cdots, f_{6}$ such that the image of the sequence $\left\{\xi_{k}\right\}$ by this combination does not tend to infinity (see definition 3.1).

Moreover, if $F$ is dominant then by Jelonek, the set $S_{F}$ has pure dimension 2 (see theorem 2.4). With this observation and with the idea of pertinent variables, we:

- Make the list $(\mathcal{L})$ of all possible façons for a polynomial mapping $F: \mathbb{C}^{3} \rightarrow \mathbb{C}^{3}$. This list is finite. In fact, there are 19 possible façons (see the list (3.4)).

- Assume that a 2-dimensional irreductible stratum $S$ of $S_{F}$ admits $l$ fixed façons in the list $(\mathcal{L})$, where $1 \leq l \leq 19$.

- Determine the pertinent variables of $F$ with respect to these $I$ façons.

- Restrict the above pertinent variables using the dominancy of $F$ and the fact that the dimension of $S$ is 2 . We get the form of $F$ in terms of these pertinent variables.

- Determine the geometry of $S$ in terms of the form of $F$.

- Let $l$ runs in the list $(\mathcal{L})$ for $1 \leq l \leq 19$. We get all the possible 2-dimensional irreductible strata of $S_{F}$. Since the dimension of $S_{F}$ is 2 , then we get the list of all possible asymptotic sets $S_{F}$.

With this idea, we provide the algorithm 3.10 to classify the asymptotic sets of dominant polynomial mappings $F: \mathbb{C}^{3} \rightarrow \mathbb{C}^{3}$ of degree 2 , and we obtain the classification theorem 4.1. This algorithm can be generalized for the general case of polynomial mappings $F: \mathbb{C}^{n} \rightarrow \mathbb{C}^{n}$ of degree $d$, where $n \geq 3$ and $d \geq 2$ 
(algorithm 5.1).

\section{Dominancy, Assymptotic Set and "Façons"}

\subsection{Dominant Polynomial Mapping}

Definition 2.1. Let $F: \mathbb{C}^{n} \rightarrow \mathbb{C}^{n}$ be a polynomial mapping. Let $\overline{F\left(\mathbb{C}^{n}\right)}$ be the closure of $F\left(\mathbb{C}^{n}\right)$ in $\mathbb{C}^{n} . F$ is called dominant if $\overline{F\left(\mathbb{C}^{n}\right)}=\mathbb{C}^{n}$, i.e., $F\left(\mathbb{C}^{n}\right)$ is dense in $\mathbb{C}^{n}$.

We provide here a lemma on the dominancy of a polynomial mapping $F: \mathbb{C}^{n} \rightarrow \mathbb{C}^{n}$ that we will use later on.

Lemma 2.2. Let $F=\left(F_{1}, \cdots, F_{n}\right): \mathbb{C}^{n} \rightarrow \mathbb{C}^{n}$ be a dominant polynomial mapping. Then, the coordinate polynomials $F_{1}, \cdots, F_{n}$ are independent. That means, there does not exist any coordinate polynomial $F_{\eta}$, where $\eta \in\{1, \cdots, n\}$, such that $F_{\eta}$ is a polynomial mapping of the variables $F_{1}, \cdots, F_{\eta-1}, F_{\eta+1}, \cdots, F_{n}$.

Proof. Assume that $F_{\eta}=\varphi\left(F_{1}, \cdots, F_{\eta-1}, F_{\eta+1}, \cdots, F_{n}\right)$ where $\eta \in\{1, \cdots, n\}$ and $\varphi$ is a polynomial. Then, the dimension of $F\left(\mathbb{C}^{n}\right)$ is less than $n$. Consequently, the dimension of $F\left(\mathbb{C}^{n}\right)$ is less than $n$. That provides the contradiction with the fact $F$ is dominant.

\subsection{Asymptotic Set}

Definition 2.3. Let $F: \mathbb{C}^{n} \rightarrow \mathbb{C}^{n}$ be a polynomial mapping. Let us denote by $S_{F}$ the set of points at which $F$ is non-proper, i.e.,

$$
S_{F}=\left\{a \in \mathbb{C}^{n} \text { such that } \exists\left\{\xi_{k}\right\}_{k \in \mathbb{N}} \subset \mathbb{C}^{n},\left|\xi_{k}\right| \rightarrow \infty \text { and } F\left(\xi_{k}\right) \rightarrow a\right\} \text {, }
$$

where $\left|\xi_{k}\right|$ is the Euclidean norm of $\xi_{k}$ in $\mathbb{C}^{n}$. The set $S_{F}$ is called the asymptotic set of $F$.

Recall that, it is sufficient to define $S_{F}$ by considering sequences $\left\{\xi_{k}\right\}$ tending to infinity in the following sense: each coordinate of these sequences either tends to infinity or converges to a finite number.

Theorem 2.4. [1] Let $F: \mathbb{C}^{n} \rightarrow \mathbb{C}^{n}$ be a polynomial mapping. If $F$ is dominant, then $S_{F}$ is either an empty set or a hypersurface.

\section{3. "Façons"}

In this section, let us recall the definition of façons as it appears in [2]. In order to a better understanding of the definition of façons, let us start by giving an example.

Example 2.5. [2] Let $F=\left(F_{1}, F_{2}, F_{3}\right): \mathbb{C}_{\left(x_{1}, x_{2}, x_{3}\right)}^{3} \rightarrow \mathbb{C}_{\left(\alpha_{1}, \alpha_{2}, \alpha_{3}\right)}^{3}$ be the polynomial mapping such that

$$
F_{1}:=x_{1}, \quad F_{2}:=x_{2}, F_{3}:=x_{1} x_{2} x_{3} .
$$

Notice that by the notations $\mathbb{C}_{\left(x_{1}, x_{2}, x_{3}\right)}^{3}$ and $\mathbb{C}_{\left(\alpha_{1}, \alpha_{2}, \alpha_{3}\right)}^{3}$, we want to distinguish the source space and the target space. We determine now the asymptotic set $S_{F}$ by using the definition 2.3. Assume that there exists a sequence $\left\{\xi_{k}=\left(x_{1, k}, x_{2, k}, x_{3, k}\right)\right\}$ in the source space $\mathbb{C}_{\left(x_{1}, x_{2}, x_{3}\right)}^{3}$ tending to infinity such that image $\left\{F\left(\xi_{k}\right)=\left(x_{1, k}, x_{2, k}, x_{1, k} x_{2, k} x_{3, k}\right)\right\}$ does not tend to infinity. Then 
$x_{1, k}$ and $x_{2, k}$ cannot tend to infinity. Since the sequence $\left\{\xi_{k}\right\}$ tends to infinity, then $x_{3, k}$ must tend to infinity. Hence, we have the three following cases:

1) $x_{1, k}$ tends to $0, x_{2, k}$ tends to a complex number $\alpha_{2} \in \mathbb{C}$ and $x_{3, k}$ tends to infinity. In order to determine the biggest possible subset of $S_{F}$, we choose the sequences $x_{1, k}$ tending to 0 and $x_{3, k}$ tending to infinity in such a way that the product $x_{1, k} x_{3, k}$ tends to a complex number $\alpha_{3}$. Let us choose, for example $\xi_{k}=\left(\frac{1}{k}, \alpha_{2}, \frac{k \alpha_{3}}{\alpha_{2}}\right)$ where $\alpha_{2} \neq 0$, then $F\left(\xi_{k}\right)$ tends to a point $a=\left(0, \alpha_{2}, \alpha_{3}\right)$ in $S_{F}$. We get a 2-dimensional stratum $S_{1}$ of $S_{F}$, where $S_{1}=\left(\alpha_{1}=0\right) \backslash$ $0 \alpha_{3} \subset \mathbb{C}_{\left(\alpha_{1}, \alpha_{2}, \alpha_{3}\right)}^{3}$. We say that a "façon" of $S_{1}$ is (3)[1]. The symbol "(3)" in the façon (3)[1] means that the third coordinate $x_{3, k}$ of the sequence $\left\{\xi_{k}\right\}$ tends to infinity. The symbol "[1]" in the façon (3)[1] means that the first coordinate $x_{1, k}$ of the sequence $\left\{\xi_{k}\right\}$ tends to 0 which is a fixed complex number which does not depend on the point $a=\left(0, \alpha_{2}, \alpha_{3}\right)$ when a describes $S_{1}$. Notice that the second coordinate of the sequence $\left\{\xi_{k}\right\}$ tends to a complex number $\alpha_{2}$ depending on the point $a=\left(0, \alpha_{2}, \alpha_{3}\right)$ when $a$ varies, then the indice " 2 " does not appear in the façon (3)[1]. Moreover, all the sequences tending to infinity such that their images tend to a point of $S_{1}$ admit only the façon (3)[1].

The two following cases are similar to the case 1 ):

2) $x_{1, k}$ tends to a complex number $\alpha_{1} \in \mathbb{C}, x_{2, k}$ tends to 0 and $x_{3, k}$ tends to infinity: then the façon (3)[2] determines a 2-dimensional stratum $S_{2}$ of $S_{F}$, where $S_{2}=\left(\alpha_{2}=0\right) \backslash 0 \alpha_{3} \subset \mathbb{C}_{\left(\alpha_{1}, \alpha_{2}, \alpha_{3}\right)}^{3}$.

3) $x_{1, k}$ and $x_{2, k}$ tend to 0 , and $x_{3, k}$ tends to infinity: then the façon (3) $[1,2]$ determines the 1-dimensional stratum $S_{3}$ where $S_{3}$ is the axis $0 \alpha_{3}$ in $\mathbb{C}_{\left(\alpha_{1}, \alpha_{2}, \alpha_{3}\right)}^{3}$.

In conclusion, we get

- the asymptotic set $S_{F}$ of the given polynomial mapping $F$ as the union of two planes $\left(\alpha_{1}=0\right)$ and $\left(\alpha_{2}=0\right)$ in $\mathbb{C}_{\left(\alpha_{1}, \alpha_{2}, \alpha_{3}\right)}^{3}$,

- all the façons of $S_{F}$ of the given polynomial mapping $F$ : they are three façons (3) [1], (3) [2] and (3) $[1,2]$.

Remark 2.6. The chosen sequence $\left\{\xi_{k}=\left(\frac{1}{k}, \alpha_{2}, \frac{k \alpha_{3}}{\alpha_{2}}\right)\right\}$ in 1) of the above example is called a generic sequence of the 2-dimensional irreductible component $\left(\alpha_{1}=0\right)$ (a plane) of $S_{F}$, since the image of any sequence of this type (with differents $\alpha_{2} \neq 0$ and $\alpha_{3}$ ) falls to a generic point of the plane $\left(\alpha_{1}=0\right)$. That means the images of all the sequences $\left\{\xi_{k}\right\}$ when $\alpha_{2}$ runs in $\mathbb{C} \backslash\{0\}$ and $\alpha_{3}$ runs in $\mathbb{C}$ cover $S_{1}=\left(\alpha_{1}=0\right) \backslash 0 \alpha_{3}$ and $S_{1}$ is dense in the plane $\left(\alpha_{1}=0\right)$. We can see easily that a generic sequence of the 2 -dimensional irreductible component $\left(\alpha_{2}=0\right)$ of $S_{F}$ is $\left(\alpha_{1}, \frac{1}{k}, \frac{k \alpha_{3}}{\alpha_{1}}\right)$ where $\alpha_{1} \neq 0$. More generally, any sequence $\left\{\left(\frac{1}{k^{r}}, \alpha_{2}, \frac{k^{r} \alpha_{3}}{\alpha_{2}}\right)\right\}$, where $r \in \mathbb{N} \backslash\{0\}$ and $\alpha_{2} \neq 0$, is a 
generic sequence of $\left(\alpha_{1}=0\right) \subset S_{F}$. Any sequence $\left\{\left(\alpha_{1}, \frac{1}{k^{r}}, \frac{k^{r} \alpha_{3}}{\alpha_{1}}\right)\right\}$, where $r \in \mathbb{N} \backslash\{0\}$ and $\alpha_{1} \neq 0$, is a generic sequence of $\left(\alpha_{2}=0\right) \subset S_{F}$.

In the light of this example, we recall here the definition of façons in [2].

Definition 2.7. [2] Let $F: \mathbb{C}^{n} \rightarrow \mathbb{C}^{n}$ be a dominant polynomial mapping such that $S_{F} \neq \varnothing$. For each point a of $S_{F}$, there exists a sequence $\left\{\xi_{k}^{a}\right\} \subset \mathbb{C}^{n}$, $\xi_{k}^{a}=\left(x_{k, 1}^{a}, \cdots, x_{k, n}^{a}\right)$ tending to infinity such that $F\left(\xi_{k}^{a}\right)$ tends to $a$. Then, there exists at least one index $i \in \mathbb{N}, 1 \leq i \leq n$ such that $x_{k, i}^{a}$ tends to infinity when $k$ tends to infinity. We define "a façon of tending to infinity of the sequence $\left\{\xi_{k}^{a}\right\}$ ", as a maximum $(p, q)$-tuple $\kappa=\left(i_{1}, \cdots, i_{p}\right)\left[j_{1}, \cdots, j_{q}\right]$ of different integers in $\{1, \cdots, n\}$, such that:

1) $x_{k, i_{r}}^{a}$ tends to infinity for all $r=1, \cdots, p$,

2) for all $s=1, \cdots, q$, the sequence $x_{k, j_{s}}^{a}$ tends to a complex number independently on the point $a$ when a varies locally, that means:

a) either there exists in $S_{F}$ a subvariety $U_{a}$ containing a such that for any point $a^{\prime}$ in $U_{a}$, there exists a sequence $\left\{\xi_{k}^{a^{\prime}}\right\} \subset \mathbb{C}^{n}, \quad \xi_{k}^{a^{\prime}}=\left(x_{k, 1}^{a^{\prime}}, \cdots, x_{k, n}^{a^{\prime}}\right)$ tending to infinity such that

i) $F\left(\xi_{k}^{a^{\prime}}\right)$ tends to $a^{\prime}$,

ii) $x_{k, i_{r}}^{a^{\prime}}$ tends to infinity for all $r=1, \cdots, p$,

iii) for all $s=1, \cdots, q, \lim _{k \rightarrow \infty} x_{k, j_{s}}^{a^{\prime}}=\lim _{k \rightarrow \infty} x_{k, j_{s}}^{a}$ and this limit is finite.

b) or there does not exist such a subvariety, then we define

$$
\kappa=\left(i_{1}, \cdots, i_{p}\right)\left[j_{1}, \cdots, j_{n-p}\right],
$$

where $x_{k, i_{r}}^{a}$ tends to infinity for all $r=1, \cdots, p$ and $\left\{i_{1}, \cdots, i_{p}\right\} \cup\left\{j_{1}, \cdots, j_{n-p}\right\}=\{1, \cdots, n\}$. In this case, the set of points $a$ is a subvariety of dimension 0 of $S_{F}$.

We call a façon of tending to infinity of the sequence $\left\{\xi_{k}^{a}\right\}$ also a a façon of a or a façon of $S_{F}$.

\section{An Algorithm to Stratify the Asymptotic Sets of the Dominant Polynominal Mappings $F: \mathbb{C}^{3} \rightarrow \mathbb{C}^{3}$ of Degree 2}

In this section we provide an algorithm to stratify the asymptotic sets associated to dominant polynominal mappings $F: \mathbb{C}^{3} \rightarrow \mathbb{C}^{3}$ of degree 2 . In the last section, we show that this algorithm can be generalized in the general case for dominant polynominal mappings $F: \mathbb{C}^{n} \rightarrow \mathbb{C}^{n}$ of degree $d$ where $n \geq 3$ and $d \geq 2$. Recall that by degree of a polynomial mapping $F=\left(F_{1}, \cdots, F_{n}\right)$ : $\mathbb{C}^{n} \rightarrow \mathbb{C}^{n}$, we mean the highest degree of the monomials $F_{1}, \cdots, F_{n}$.

Let us consider now a dominant polynomial mapping $F=\left(F_{1}, F_{2}, F_{3}\right)$ : $\mathbb{C}_{\left(x_{1}, x_{2}, x_{3}\right)}^{3} \rightarrow \mathbb{C}_{\left(\alpha_{1}, \alpha_{2}, \alpha_{3}\right)}^{3}$ of degree 2 such that $S_{F} \neq \varnothing$. An important step of this section is to define the notion of "pertinent" variables of $F$.

\subsection{Pertinent Variables}

Let us explain at first the idea of the notion of pertinent variables: let $\left\{\xi_{k}\right\}=$ 
$\left\{\left(x_{1, k}, x_{2, k}, x_{3, k}\right)\right\}$ be a sequence in the source space $\mathbb{C}_{\left(x_{1}, x_{2}, x_{3}\right)}^{3}$ tending to infinity such that $F\left(\xi_{k}\right)$ tends to a point of $S_{F}$ in the target space $\mathbb{C}_{\left(\alpha_{1}, \alpha_{2}, \alpha_{3}\right)}^{3}$. Then the image of $\xi_{k}$ by any coordinate polynomial $F_{\eta}$, where $\eta=1,2,3$, cannot tend to infinity. Notice that $F_{\eta}$ can be written as the sum of elements of the form $F_{\eta}^{1} F_{\eta}^{2}$ such that if $F_{\eta}^{1}\left(\xi_{k}\right)$ tends to infinity, then $F_{\eta}^{2}\left(\xi_{k}\right)$ must tend to 0 . In other words, if one element of the above sum has a factor tending to infinity with respect to the sequence $\left\{\xi_{k}\right\}$, then this element must be "balanced" with another factor tending to zero with respect to the sequence $\left\{\xi_{k}\right\}$. For example, assume that the coordinate sequences $x_{1, k}$ and $x_{2, k}$ of the sequence $\left\{\xi_{k}\right\}$ tend to infinity, then $F_{\eta}$ cannot admit neither $x_{1}$ nor $x_{2}$ alone as an element of the above sum, but $F_{\eta}$ can admit $\left(x_{1}-v x_{2}\right)$, $\left(x_{1}-v x_{2}\right) x_{1},\left(x_{1}-v x_{2}\right) x_{2}$ as elements of this sum, where $v \in \mathbb{C} \backslash\{0\}$. So we define:

Definition 3.1. Let $F=\left(F_{1}, F_{2}, F_{3}\right): \mathbb{C}_{\left(x_{1}, x_{2}, x_{3}\right)}^{3} \rightarrow \mathbb{C}_{\left(\alpha_{1}, \alpha_{2}, \alpha_{3}\right)}^{3}$ be a polynomial mapping of degree 2 such that $S_{F} \neq \varnothing$. Let us fix a façon $\kappa$ of $S_{F}$. Then there exists a sequence $\left\{\xi_{k}\right\} \subset \mathbb{C}_{\left(x_{1}, x_{2}, x_{3}\right)}^{3}$ tending to infinity with the façon $\kappa$ such that its image tend to a point in $S_{F}$. An element in the list

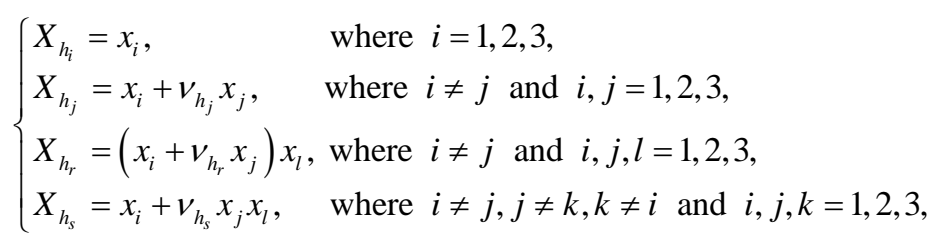

$\left(v_{h_{i}}, v_{h_{j}}, v_{h_{r}}, v_{h_{s}} \in \mathbb{C} \backslash\{0\}\right)$ is called a pertinent variable of $F$ with respect to the façon $\kappa$ if the image of the sequence $\left\{\xi_{k}\right\}$ by this element does not tend to infinity.

Remark 3.3. From now on, we will denote $X_{1}, \cdots, X_{h}$ pertinent variables of $F$ with respect to a fixed façon and we write

$$
F=\tilde{F}\left(X_{1}, \cdots, X_{h}\right) .
$$

Notice that we can also determine the pertinent variables of $F$ with respect to a set of façons in the case we have more than one façon.

\subsection{Idea of the Algorithm}

The aim of the algorithm that we present in this section is to describe the list of all possible asymptotic sets $S_{F}$ for the dominant polynomial mappings $F: \mathbb{C}^{3} \rightarrow \mathbb{C}^{3}$ of degree 2 . In order to do that, we observe firstly that

- The list of all the possible façons of $S_{F}$ for a polynomial mapping

$$
F: \mathbb{C}^{3} \rightarrow \mathbb{C}^{3} \text { is }
$$

$$
\left\{\begin{array}{l}
\text { 1) GroupI : }(1,2,3), \\
\text { 2) GroupII :(1,2),(2,3) and }(3,1), \\
\text { 3) GroupIII : }(1),(2) \text { and }(3), \\
\text { 4) GroupIV :(1,2)[3],(1,3)[2] and }(2,3)[1], \\
\text { 5)GroupV :(1)[2],(1)[3],(2)[1],[2](3),[3](1) and [3](2), } \\
\text { 6)GroupVI:(1)[2,3],(2)[1,3] and }(3)[1,2] .
\end{array}\right.
$$


This list has 19 façons.

- Since $F$ dominant, then by the theorem 2.4 , the set $S_{F}$ has pure dimen- sion 2.

With these observations, we will:

- assume that a 2-dimensional irreductible stratum $S$ of $S_{F}$ admits 1 fixed façons in the list (3.4), where $1 \leq l \leq 19$,

- determine the pertinent variables of $F$ with respect to these 1 façons,

- restrict the above pertinent variables by using the dominancy of $F$ and the fact $\operatorname{dim} S=2$. We get the form of $F$ in terms of these pertinent variables,

- determine the geometry of $S$ in terms of the form of $F$,

- let $I$ run in the list (3.4) for $1 \leq l \leq 19$. We get all the possible 2-dimen- sional irreductible strata $S$ of $S_{F}$. Since the dimension of $S_{F}$ is 2 , then we get the list of all the possible asymptotic sets $S_{F}$ of $F$.

The following example explains the process of the algorithm, i.e. how we can determine the geometry of a 2-dimensional irreductible stratum $S$ of $S_{F}$ admitting some fixed façons.

\subsection{Example}

Example 3.5. Let $F: \mathbb{C}^{3} \rightarrow \mathbb{C}^{3}$ be a dominant polynomial mapping of degree 2. Assume that a 2-dimensional stratum $S$ of $S_{F}$ admits the two façons $\kappa=(1,2,3)$ and $\kappa^{\prime}=(1,2)[3]$. That means that all the sequences tending to infinity in the source space such that their images tend to the points of $S$ admit either the façon $\kappa=(1,2,3)$ or the façon $\kappa^{\prime}=(1,2)[3]$. In order to describe the geometry of $S$, we perform the following steps:

Step 1: Determine the pertinent variable of $F$ with respect to the façons $\kappa=(1,2,3)$ and $\kappa^{\prime}=(1,2)[3]$ :

- With the façon $\kappa=(1,2,3)$, all the three coordinate sequences of the corresponding sequence tend to infinity ( $c f$. Definition 2.7 ). Up to a suiable linear change of coordinates, the mapping $F$ admits the pertinent variables: $x_{1}-x_{2},\left(x_{1}-x_{2}\right) x_{1},\left(x_{1}-x_{2}\right) x_{2},\left(x_{1}-x_{2}\right) x_{3}, x_{1}-x_{3},\left(x_{1}-x_{3}\right) x_{1}$, $\left(x_{1}-x_{3}\right) x_{2},\left(x_{1}-x_{3}\right) x_{3}, x_{2}-x_{3},\left(x_{2}-x_{3}\right) x_{1},\left(x_{2}-x_{3}\right) x_{2},\left(x_{2}-x_{3}\right) x_{3}$, $x_{1}-x_{2} x_{3}, x_{2}-x_{1} x_{3}$ and $x_{3}-x_{1} x_{2}$ (see definition 3.1).

- With the façon $\kappa^{\prime}=(1,2)[3]$, the first and second coordinate sequences of the corresponding sequence tend to infinity, the third coordinate sequence of the corresponding sequence tends to a fixed complex number. As we refer to the same mapping $F$, then up to the same suiable linear change of coordinates, the mapping $F$ admits the pertinent variables: $x_{3}, x_{1} x_{3}, x_{2} x_{3}$, $x_{1}-x_{2},\left(x_{1}-x_{2}\right) x_{1},\left(x_{1}-x_{2}\right) x_{2},\left(x_{1}-x_{2}\right) x_{3}$ and $\left(x_{1}-x_{3}\right) x_{3}$.

Since $S$ contains both of the façons $\kappa$ and $\kappa^{\prime}$, then this surface $S$ admits $x_{1}-x_{2},\left(x_{1}-x_{2}\right) x_{1},\left(x_{1}-x_{2}\right) x_{2},\left(x_{1}-x_{2}\right) x_{3}$ and $\left(x_{1}-x_{3}\right) x_{3}$ as pertinent variables. Let us denote by

$$
\begin{aligned}
& X_{1}=x_{1}-x_{2}, X_{2}=\left(x_{1}-x_{2}\right) x_{1}, X_{3}=\left(x_{1}-x_{2}\right) x_{2}, \\
& X_{4}=\left(x_{1}-x_{2}\right) x_{3}, X_{5}=\left(x_{1}-x_{3}\right) x_{3} .
\end{aligned}
$$


We can write

$$
F=\tilde{F}\left(X_{1}, X_{2}, X_{3}, X_{4}, X_{5}\right) .
$$

Step 2: Assume that $\left\{\xi_{k}=\left(x_{1, k}, x_{2, k}, x_{3, k}\right)\right\}$ and $\left\{\xi_{k}^{\prime}=\left(x_{1, k}^{\prime}, x_{2, k}^{\prime}, x_{3, k}^{\prime}\right)\right\}$ are two sequences tending to infinity with the façons $\kappa$ and $\kappa^{\prime}$, respectively.

A) Let us consider the façon $\kappa=(1,2,3)$ and its corresponding generic sequence $\left\{\xi_{k}=\left(x_{1, k}, x_{2, k}, x_{3, k}\right)\right\}$ :

- Assume that $X_{1}\left(\xi_{k}\right)=\left(x_{1, k}-x_{2, k}\right)$ tends to a non-zero complex number. Since $\kappa=(1,2,3)$ then all three coordinate sequences $x_{1, k}, x_{2, k}$ and $x_{3, k}$ tend to infinity. Hence $X_{2}\left(\xi_{k}\right)=\left(x_{1, k}-x_{2, k}\right) x_{1, k}, X_{3}\left(\xi_{k}\right)=\left(x_{1, k}-x_{2, k}\right) x_{2, k}$ and $X_{4}\left(\xi_{k}\right)=\left(x_{1, k}-x_{2, k}\right) x_{3, k}$ tend to infinity. In this case, $X_{2}, X_{3}$ and $X_{4}$ cannot be pertinent variables of $F$ anymore. Then $F$ admits only two pertinent variables $X_{1}$ and $X_{5}$, or $F=\tilde{F}\left(X_{1}, X_{5}\right)$. We can see that the dimension of $S$ in this case is 1 , that provides a contradiction with the fact that the dimension of $S$ is 2 . Consequently, $\left(x_{1, k}-x_{2, k}\right)$ tends to 0 .

- Assume that $\left(x_{1, k}-x_{3, k}\right)$ tends to a non-zero complex number. Then $X_{5}\left(\xi_{k}\right)=\left(x_{1, k}-x_{3, k}\right) x_{3, k}$ tend to infinity, hence $X_{5}$ cannot be a pertinent variable of $F$ anymore, then $F=\tilde{F}\left(X_{1}, X_{2}, X_{3}, X_{4}\right)$. We choose a generic sequence $\left\{\xi_{k}\right\}$ satisfying the conditions: $X_{1, k}=\left(x_{1, k}-x_{2, k}\right)$ tends to zero and $\left(x_{1, k}-x_{3, k}\right)$ tends to a non-zero complex number, for example, $\xi_{k}=$ $(k+\alpha / k, k+\beta / k, k+\gamma)$. Then $\quad X_{2}\left(\xi_{k}\right)=\left(x_{1, k}-x_{2, k}\right) x_{1, k}, \quad X_{3}\left(\xi_{k}\right)=$ $\left(x_{1, k}-x_{2, k}\right) x_{2, k}$ and $X_{4}\left(\xi_{k}\right)=\left(x_{1, k}-x_{2, k}\right) x_{3, k}$ tend to the same complex number $\lambda-\mu$. Combining with the fact $X_{1, k}=\left(x_{1, k}-x_{2, k}\right)$ tends to zero, we conclude that the dimension of $S$ in this case is 1 , that provides a contradiction with the fact that the dimension of $S$ is 2. Consequently, $\left(x_{1, k}-x_{3, k}\right)$ tends to 0 .

Then, with the façon $\kappa$, we have $\left(x_{1, k}-x_{2, k}\right)$ and $\left(x_{1, k}-x_{3, k}\right)$ tend to 0 . Hence $\left(x_{2, k}-x_{3, k}\right)$ also tends to 0 . Let us choose a generic sequence $\left\{\xi_{k}\right\}$ satisfying these conditions, for example, the sequence $\left\{\xi_{k}=(k+\alpha / k, k+\beta / k, k+\gamma / k)\right\}$. We see that $X_{2, k}=\left(x_{1, k}-x_{2, k}\right) x_{1, k}$, $X_{3, k}=\left(x_{1, k}-x_{2, k}\right) x_{2, k}$ and $X_{4, k}=\left(x_{1, k}-x_{2, k}\right) x_{3, k}$ tend to a same complex number $\lambda=\alpha-\beta$. Moreover, $X_{5, k}=\left(x_{1, k}-x_{3, k}\right) x_{3, k}$ tends to $\mu=\alpha-\gamma$. So we have

$$
\lim _{k \rightarrow \infty} F\left(\xi_{k}\right)=\tilde{F}(0, \lambda, \lambda, \lambda, \mu)
$$

B) Let us consider now the façon $\kappa^{\prime}=(1,2)[3]$ and its corresponding generic sequence $\left\{\xi_{k}^{\prime}=\left(x_{1, k}^{\prime}, x_{2, k}^{\prime}, x_{3, k}^{\prime}\right)\right\}$, we have two cases:

- If $X_{1}\left(\xi_{k}^{\prime}\right)=\left(x_{1, k}^{\prime}-x_{2, k}^{\prime}\right)$ tends to 0: So $X_{4}\left(\xi_{k}^{\prime}\right)=\left(x_{1, k}^{\prime}-x_{2, k}^{\prime}\right) x_{3, k}^{\prime}$ tends to 0 . We have $X_{2}\left(\xi_{k}^{\prime}\right)=\left(x_{1, k}^{\prime}-x_{2, k}^{\prime}\right) x_{1, k}^{\prime}$ and $X_{2}\left(\xi_{k}^{\prime}\right)=\left(x_{1, k}^{\prime}-x_{2, k}^{\prime}\right) x_{2, k}^{\prime}$ tend to a same complex number $\lambda^{\prime}$ and $X_{5}\left(\xi_{k}^{\prime}\right)=\left(x_{1, k}^{\prime}-x_{3, k}^{\prime}\right) x_{3, k}^{\prime}$ tends to an arbitrary complex number $\mu^{\prime}$. Then in this case, we have

$$
F\left(\xi_{k}^{\prime}\right)=\tilde{F}\left(0, \lambda^{\prime}, \lambda^{\prime}, 0, \mu^{\prime}\right) \text {. }
$$

- If $X_{1}\left(\xi_{k}^{\prime}\right)=\left(x_{1, k}^{\prime}-x_{2, k}^{\prime}\right)$ tends to a non-zero complex number $\lambda^{\prime} \in \mathbb{C}$ : So $X_{2}\left(\xi_{k}^{\prime}\right)$ and $X_{3}\left(\xi_{k}\right)$ tend to infinity, thus $X_{2}$ and $X_{3}$ cannot be 
pertinent variables of $F$ anymore. Moreover, $X_{4}\left(\xi_{k}^{\prime}\right)$ tends to 0 and $X_{5}\left(\xi_{k}^{\prime}\right)$ tends to an arbitrary complex number $\mu^{\prime}$. Then in this case, we have

$$
\begin{aligned}
& F=\tilde{F}\left(X_{1}, X_{4}, X_{5}\right) \\
& F\left(\xi_{k}^{\prime}\right)=\tilde{F}\left(\lambda^{\prime}, 0, \mu^{\prime}\right) .
\end{aligned}
$$

In conclusion, we have two cases:

1) From (3.6), (3.7) and (3.8), we have

$$
\begin{aligned}
& F=\tilde{F}\left(X_{1}, X_{2}, X_{3}, X_{4}, X_{5}\right) \\
& \lim _{k \rightarrow \infty} F\left(\xi_{k}\right)=\tilde{F}(0, \lambda, \lambda, \lambda, \mu) \\
& \lim _{k \rightarrow \infty} F\left(\xi_{k}^{\prime}\right)=\tilde{F}\left(0, \lambda^{\prime}, \lambda^{\prime}, 0, \mu^{\prime}\right) .
\end{aligned}
$$

2) From (3.6), (3.7) and (3.9), we have

$$
\begin{aligned}
& F=\tilde{F}\left(X_{1}, X_{4}, X_{5}\right) \\
& \lim _{k \rightarrow \infty} F\left(\xi_{k}\right)=\tilde{F}(0, \lambda, \mu) \\
& \lim _{k \rightarrow \infty} F\left(\xi_{k}^{\prime}\right)=\tilde{F}\left(\lambda^{\prime}, 0, \mu^{\prime}\right) .
\end{aligned}
$$

Step 3: We restrict the pertinent variables in the step 2 by using the three following facts:

- $\kappa$ and $\kappa^{\prime}$ are two façons of the same stratum $S$,

- $\operatorname{dim} S=2$,

- $F$ is dominant.

Let us consider the two cases $\left({ }^{*}\right)$ and $\left({ }^{*}\right)$ determined in the step 2:

1) $F$ is of the form $\left(^{*}\right)$ :

- At first, we use the fact that $\kappa$ and $\kappa^{\prime}$ are two façons of the same stratum $S$, then if $X_{i}$ is a pertinent variable of $F$ then both $X_{i}\left(\xi_{k}\right)$ and $X_{i}\left(\xi_{k}^{\prime}\right)$ must tend to either an arbitrary complex number or zero.

- Since the dimension of $S$ is 2 then $F$ must have at least two pertinent variables $X_{i}$ and $X_{j}$ such that the images of the sequences $\xi_{k}$ and $\xi_{k}^{\prime}$ by $X_{i}$ and $X_{j}$, respectively, tend independently to two complex numbers. In this case:

$+F$ must admit either $X_{2}=\left(x_{1}-x_{2}\right) x_{1}$ or $X_{3}=\left(x_{1}-x_{2}\right) x_{2}$ as a pertinent variable,

$+F$ must admit $X_{5}=\left(x_{1}-x_{3}\right) x_{3}$ as a pertinent variable.

- Since $F$ is dominant then $F$ must admit at least 3 independent pertinent variables (see lemma 2.2). Then in this case, $F$ must also admit $X_{1}=x_{1}-x_{2}$ as a pertinent variable. We see that $X_{1}\left(\xi_{k}\right)$ and $X_{1}\left(\xi_{k}^{\prime}\right)$ tend to 0 . We can say that this variable is a "free" pertinent variable. The role of this variable is to guarantee the fact that $F\left(\mathbb{C}^{3}\right)$ is dense in the target space $\mathbb{C}^{3}$.

2) $F$ is of the form (**): Similarly to the case 1 , we can see easily that $F$ can admit only $X_{5}$ as a pertinent variable. Then the dimension of $S$ is 1 , which is a contradiction with the fact that the dimension of $S$ is 2 . 
In conclusion, $F$ has the following form:

$$
\begin{aligned}
& F=\tilde{F}\left(X_{1}, X_{2}, X_{3}, X_{5}\right) \\
& \lim _{k \rightarrow \infty} F\left(\xi_{k}\right)=\tilde{F}(0, \lambda, \lambda, \mu) \\
& \lim _{k \rightarrow \infty} F\left(\xi_{k}^{\prime}\right)=\tilde{F}\left(0, \lambda^{\prime}, \lambda^{\prime}, \mu^{\prime}\right) .
\end{aligned}
$$

Step 4: Describe the geometry of the 2-dimensional stratum $S$ : On the one hand, the pertinent variables $X_{2}$ (or $X_{3}$ ) and $X_{5}$ tending independently to two complex numbers have degree 2; on the other hand, the degree of $F$ is 2, then the degree of the surface $S$ with respect to the variables $\lambda$ and $\mu$ (or $\lambda^{\prime}$ and $\mu^{\prime}$ ) is 1 (notice that by degree of $S$, we mean the degree of the equation defining $S$ ). We conclude that $S$ is a plane.

In light of the example 3.5, we explicit now the algorithm for classifying the asymptotic sets of the non-proper dominant polynomial mappings

$F=\left(F_{1}, F_{2}, F_{3}\right): \mathbb{C}^{3} \rightarrow \mathbb{C}^{3}$ of degree 2.

\subsection{Algorithm}

Algorithm 3.10. We have the five following steps:

\section{Step 1:}

- Fix $l$ façons $\kappa_{1}, \cdots, \kappa_{l}$ in the list (3.4), where $1 \leq l \leq 19$.

- Determine the pertinent variables with respect to these $l$ façons (knowing that they must be refered to a same mapping $F$ ).

\section{Step 2:}

- Assume that $S$ is a 2-dimensional stratum of $S_{F}$ admitting only the $I$ façons $\kappa_{1}, \cdots, \kappa_{l}$ in step 1 .

- Take generic sequences $\xi_{k}^{1}, \cdots, \xi_{k}^{l}$ corresponding to $\kappa_{1}, \cdots, \kappa_{l}$, respectively.

- Compute the limit of the images of the sequences $\xi_{k}^{1}, \cdots, \xi_{k}^{l}$ by the pertinent variables defined in step 1.

- Restrict the pertinent variables in step 1 using the fact $\operatorname{dim} S=2$.

Step 3: Restrict again the pertinent variables in step 2 using the three following facts:

- the façons $\kappa_{1}, \cdots, \kappa_{l}$ belongs to $S$ : then the images of the generic sequences $\xi_{k}^{1}, \cdots, \xi_{k}^{l}$ by the pertinent variables defined in the step 2 must tend to either an arbitrary complex number or zero,

- $\operatorname{dim} S=2$ : then there are at least two pertinent variables $X_{i}$ and $X_{j}$ such that the images of the sequences $\xi_{k}$ and $\xi_{k}^{\prime}$ by $X_{i}$ and $X_{j}$, respectively, tend independently to two complex numbers,

- $F$ is dominant: then there are at least 3 independent pertinent variables (see lemma 2.2).

Step 4: Describe the geometry of the 2-dimensional irreductible stratum $S$ of $S_{F}$ in terms of the pertinent variables obtained in the step 3.

Step 5: Letting 1 run from 1 to 19 in the list (3.4).

Theorem 3.11. With the algorithm 3.10, we obtain the list of all possible asymptotic sets $S_{F}$ of non-proper dominant polynomial mappings $F: \mathbb{C}^{3} \rightarrow \mathbb{C}^{3}$ of degree 2 . 
Proof. On the one hand, the process of the algorithm 3.10 is possible, since the number of the façons in the list (3.4) is finite (19 façons). On the other hand, by the step 2, step 4 and step 5, we consider all the possible cases for all 2-dimensional irreductible strata of $S_{F}$. Since the dimension of $S_{F}$ is 2 (see theorem 2.4), we get all the possible asymptotic sets $S_{F}$ of non-proper dominant polynomial mappings $F: \mathbb{C}^{3} \rightarrow \mathbb{C}^{3}$ of degree 2 .

\section{Results}

In this section, we use the algorithm 3.10 to prove the following theorem.

Theorem 4.1. The asymptotic set of a non-proper dominant polynomial mapping $F: \mathbb{C}^{3} \rightarrow \mathbb{C}^{3}$ of degree 2 is one of the five elements in the following list $\mathcal{L}_{S_{F}}^{(3,2)}$. Moreover, any element of this list can be realized as the asymptotic set of a dominant polynomial mapping $F: \mathbb{C}^{3} \rightarrow \mathbb{C}^{3}$ of degree 2 .

The list $\mathcal{L}_{S_{F}}^{(3,2)}$ :

1) A plane.

2) A paraboloid.

3) The union of a plane $(\mathscr{T}): r_{1} x_{1}+r_{2} x_{2}+r_{3} x_{3}+r_{4}=0$, and a plane of the form $\left(\mathscr{P}^{\prime}\right): r_{1}^{\prime} x_{1}+r_{2}^{\prime} x_{2}+r_{3}^{\prime} x_{3}+r_{4}^{\prime}=0$, where we can choose two of the three coefficients $r_{1}^{\prime}, r_{2}^{\prime}, r_{3}^{\prime}$, then the third of them and the fourth coefficient $r_{4}^{\prime}$ are determined.

4) The union of a plane $(\mathscr{P}): r_{1} x_{1}+r_{2} x_{2}+r_{3} x_{3}+r_{4}=0$ and a paraboloid of the form $(\mathscr{C}): r_{i}^{\prime} x_{i}^{2}+r_{j}^{\prime} x_{j}+r_{l}^{\prime} x_{l}+r_{4}^{\prime}=0,\{i, j, l\}=\{1,2,3\}$, where we can choose two of the three coefficients $r_{1}^{\prime}, r_{2}^{\prime}, r_{3}^{\prime}$, then the third of them and the fourth coefficient $r_{4}^{\prime}$ are determined.

5) The union of three planes

$$
\begin{aligned}
& (\mathscr{P}): r_{1} x_{1}+r_{2} x_{2}+r_{3} x_{3}+r_{4}=0, \\
& \left(\mathscr{P}^{\prime}\right): r_{1}^{\prime} x_{1}+r_{2}^{\prime} x_{2}+r_{3}^{\prime} x_{3}+r_{4}^{\prime}=0, \\
& \left(\mathscr{P}^{\prime \prime}\right): r_{1}^{\prime \prime} x_{1}+r_{2}^{\prime \prime} x_{2}+r_{3}^{\prime \prime} X_{3}+r_{4}^{\prime \prime}=0,
\end{aligned}
$$

where:

a) for $\left(\mathscr{P}^{\prime}\right)$, we can choose two of the three coefficients $r_{1}^{\prime}, r_{2}^{\prime}, r_{3}^{\prime}$, then the third of them and the fourth coefficient $r_{4}^{\prime}$ are determined,

b) for $\left(\mathscr{P}^{\prime \prime}\right)$, we can choose two of the three coefficients $r_{1}^{\prime \prime}, r_{2}^{\prime \prime}, r_{3}^{\prime \prime}$, then the third of them and the fourth coefficient $r_{4}^{\prime \prime}$ are determined.

In order to prove this theorem, we need the two following lemmas.

Lemma 4.2. Let $F=\left(F_{1}, F_{2}, F_{3}\right): \mathbb{C}^{3} \rightarrow \mathbb{C}^{3}$ be a non-proper dominant polynomial mapping of degree 2. If $S_{F}$ contains a surface of degree higher than 1, then either $S_{F}$ is a paraboloid, or $S_{F}$ is the union of a paraboloid and a plane.

Proof. Assume that $S_{F}$ contains a surface $(\mathscr{C})$. Since $\operatorname{deg} F=2$ then $1 \leq \operatorname{deg}(\mathscr{H}) \leq 2$.

A) We prove firstly that if $S_{F}$ contains a surface $(\mathscr{C})$ where $\operatorname{deg}(\mathscr{C})=2$ then $(\mathscr{C})$ is a paraboloid. Since $\operatorname{deg}(\mathscr{C})=2$ and $\operatorname{deg} F=2$ then $S_{F}$ admits one façon $\kappa$ in such a way that among the pertinent variables of $F$ with 
respect to the façon $\kappa$, there exists only one free pertinent variable. That means, one of $x_{1}, x_{2}$ and $x_{3}$ is a pertinent variable of $F$ with respect to $\kappa$ (cf. Definition 3.1). Without loose of generality, we assume that $x_{1}$ is a pertinent variable of $F$ with respect to the façon $\kappa=(3)$ [2]. Assume that $\left\{\xi_{k}=\left(x_{1, k}, x_{2, k}, x_{3, k}\right)\right\}$ is a generic sequence tending to infinity with the façon $\kappa$ and

i) $x_{3, k}$ tends to infinity, $x_{2, k}$ tends to 0 in such a way that $x_{2, k} x_{3, k}$ tends to an arbitrary complex number $\lambda$,

ii) $x_{1, k}$ tends to an arbitrary complex number $\mu$.

We see that $x_{1, k}^{2}$ and $\left(x_{1, k}+x_{2, k}\right) x_{1, k}$ tend to $\mu^{2}$. Since $\operatorname{deg} F=2$ and $\operatorname{deg}(\mathscr{C})=2$, then

i) one coordinate polynomial $F_{\eta}$, where $\eta \in\{1,2,3\}$, must contain $x_{1}$ as an element of degree 1 ,

ii) the another coordinate polynomial $F_{\eta^{\prime}}$, where $\eta^{\prime} \in\{1,2,3\}$ and $\eta^{\prime} \neq \eta$, must contain $x_{1}^{2}$ or $\left(x_{1}+x_{2}\right) x_{1}$ as a pertinent variable.

Assume that the equation of the surface $(\mathscr{C})$ is $r_{1} \alpha_{1}^{p_{1}}+r_{2} \alpha_{2}^{p_{2}}+$ $r_{3} \alpha_{3}^{p_{3}}+r_{4}=0$. Since $x_{1, k}^{2}$ and $\left(x_{1, k}+x_{2, k}\right) x_{1, k}$ tend to the same complex number $\mu^{2}$, and $\operatorname{deg}(\mathscr{C})=2$, then there exists an unique index $i \in\{1,2,3\}$ such that $r_{i} \neq 0$ and $p_{i}=2$. If $r_{j}=0$ or $p_{j}=0$ for all $j \neq i, j \in\{1,2,3\}$, then $(\mathscr{H})$ is the union of two lines. That provides the contradiction with the fact that $\operatorname{deg}(\mathscr{C})=2$. So, there exists $j \neq i, j \in\{1,2,3\}$ such that $r_{j} \neq 0$ and $p_{j}=1$. Consequently, the surface $(\mathscr{C})$ is a paraboloid.

B) We prove now that if $S_{F}$ contains a paraboloid then the biggest possible $S_{F}$ is the union of this paraboloid and a plane. Since $S_{F}$ contains a paraboloid then with the same choice of the façon $\kappa=(3)[2]$ as in A), the mapping $F$ must be considered as a dominant polynomial mapping of pertinent variables $x_{1}, x_{2}, x_{1} x_{2}$ and $x_{2} x_{3}$, that means:

$$
F=\tilde{F}\left(x_{1}, x_{2}, x_{1} x_{2}, x_{2} x_{3}\right) .
$$

We can see easily that if $x_{2}$ is a pertinent variable of $F$, then $S_{F}$ admits only the façon $\kappa$ and $S_{F}$ is a paraboloid. Assume that $S_{F}$ contains another irreductible surface $\left(\mathscr{C}^{\prime}\right)$ which is different from $(\mathscr{C})$. Then $F$ must be considered as a polynomial mapping of pertinent variables $x_{1}, x_{1} x_{2}$ and $x_{2} x_{3}$, that means:

$$
F=\tilde{F}\left(x_{1}, x_{1} x_{2}, x_{2} x_{3}\right) .
$$

Let us consider now one façon $\kappa^{\prime}$ of $\left(\mathscr{C}^{\prime}\right)$ such that $\kappa^{\prime} \neq \kappa$ and let $\left\{\xi_{k}^{\prime}=\left(x_{1, k}^{\prime}, x_{2, k}^{\prime}, x_{3, k}^{\prime}\right)\right\}$ be a corresponding generic sequence of $\kappa^{\prime}$. Notice that one coordinate of $F$ admits $x_{1}$ as a pertinent variable. Let us show that $x_{1, k}^{\prime}$ tends to 0 . Assume that $x_{1, k}^{\prime}$ tends to a non-zero complex number. As one coordinate of $F$ admits $\quad x_{1} x_{2}$ as a pertinent variable, then $x_{2, k}^{\prime}$ does not tend to infinity. We have two cases:

+ If $x_{2, k}^{\prime}$ tends to 0 , then in order to $\xi_{k}^{\prime}$ tending to infinity, $x_{3, k}^{\prime}$ must tend to infinity. Hence, the façon $\kappa^{\prime}$ is (3)[2]. That provides the contradiction with the fact $\kappa^{\prime} \neq \kappa$. 
+ If $x_{2, k}^{\prime}$ tends to a non-zero finite complex number, since one coordinate of $F$ admits $x_{2} x_{3}$ as factor, then $x_{3, k}^{\prime}$ does not tend to infinity. That provides the contradiction with the fact that $\xi_{k}^{\prime}$ tends to infinity.

Therefore, $x_{1, k}^{\prime}$ tends to 0 . We have the following possible cases:

1) $\kappa^{\prime}=(2)[1]$ : then $F$ is a polynomial mapping of the form $F=\tilde{F}\left(x_{1}, x_{3}, x_{1} x_{2}, x_{1} x_{3}\right)$. Combining with (4.3), then $F=\tilde{F}\left(x_{1}, x_{1} x_{2}\right)$. Therefore, $F$ is not dominant, which provides the contradiction.

2) $\kappa^{\prime}=(3)[1]$ : then $F$ is a polynomial mapping of the form $F=\tilde{F}\left(x_{1}, x_{2}, x_{1} x_{2}, x_{1} x_{3}\right)$. Combining with (4.3), then $F=\tilde{F}\left(x_{1}, x_{1} x_{2}\right)$. Therefore, $F$ is not dominant, which provides the contradiction.

3) $\boldsymbol{\kappa}^{\prime}=(2,3)[1]$ : then $F$ is a polynomial mapping of the form $F=\tilde{F}\left(x_{1}, x_{1} x_{2}, x_{1} x_{3}\right)$. Combining with (4.3), then $F=\tilde{F}\left(x_{1}, x_{1} x_{2}\right)$. Therefore, $F$ is not dominant, which provides the contradiction.

4) $\kappa^{\prime}=(3)[1,2]$ : then $F=\tilde{F}\left(x_{1}, x_{2}, x_{1} x_{2}, x_{2} x_{3}, x_{3} x_{1}\right)$. Combining with (4.3), we have $F=\tilde{F}\left(x_{1}, x_{1} x_{2}, x_{2} x_{3}\right)$. Since $x_{1, k}^{\prime} x_{2, k}^{\prime}$ and $x_{1, k}^{\prime}$ tend to 0 , then $\operatorname{dim}\left(\mathscr{C}^{\prime}\right) \leq 1$, that provides the contradiction.

5) $\kappa^{\prime}=(2)[1,3]$ : in this case, $F$ is a polynomial mapping admitting the form

$$
F=\tilde{F}\left(x_{1}, x_{3}, x_{1} x_{2}, x_{2} x_{3}, x_{3} x_{1}\right) .
$$

Combining with (4.3), then

$$
F=\tilde{F}\left(x_{1}, x_{1} x_{2}, x_{2} x_{3}\right) .
$$

We know that $x_{1, k}^{\prime}$ tends to 0 . Assume that $x_{1, k}^{\prime} x_{2, k}^{\prime}$ tends to a complex number $\lambda$ and $x_{2, k}^{\prime} x_{3, k}^{\prime}$ tends to a complex number $\mu$, we have

$$
\left(\mathscr{C}^{\prime}\right)=\left\{\left(\tilde{F}_{1}(0, \lambda, \mu), \tilde{F}_{2}(0, \lambda, \mu), \tilde{F}_{3}(0, \lambda, \mu)\right): \lambda, \mu \in \mathbb{C}\right\},
$$

where $\tilde{F}=\left(\tilde{F}_{1}, \tilde{F}_{2}, \tilde{F}_{3}\right)$. Since $\operatorname{deg} F=2$, then the degree of $\tilde{F}_{i}$ with respect to the variables $\lambda$ and $\mu$ must be 1 , for all $i \in\{1,2,3\}$. Consequently, the surface $\left(\mathscr{H}^{\prime}\right)$ is a plane.

Lemma 4.4. Let $F: \mathbb{C}^{3} \rightarrow \mathbb{C}^{3}$ be a non-proper dominant polynomial mapping of degree 2. Assume that $S$ is a 2-dimensional irreductible stratum of $S_{F}$. Then $S$ admits at most two façons. Moreover, if $S$ admits two façons, then $S_{F}$ is a plane.

Proof. Let $F: \mathbb{C}^{3} \rightarrow \mathbb{C}^{3}$ be a non-proper dominant polynomial mapping of degree 2. Assume that $S$ is a 2-dimensional irreductible stratum of $S_{F}$.

A) We provide firstly the list of pairs of façons that $S$ can admit and we write $F$ in terms of pertinent variables in each of these cases. Let us fix a pair of façons $\left(\kappa, \kappa^{\prime}\right)$ in the list (3.4) and assume that $S$ admits these two façons. We use the steps 1, 2, 3 and 4 of the algorithm 3.10. In the same way than the example 3.5, we can determine the form of $F$ in terms of its pertinent variables with respect to two fixed façons after using the conditions of dimension of $S$ and the dominancy of $F$. Letting two façons $\kappa, \kappa^{\prime}$ run in the list (3.4), we get the following possiblilities:

1) $\left(\kappa, \kappa^{\prime}\right)=\left((1,2,3),\left(i_{1}, i_{2}\right)[j]\right)$, where $\left\{i_{1}, i_{2}, j\right\}=\{1,2,3\}$ and

$$
F=\tilde{F}\left(x_{i_{1}}-x_{i_{2}},\left(x_{i_{1}}-x_{i_{2}}\right) x_{i_{1}},\left(x_{i_{1}}-x_{i_{2}}\right) x_{i_{2}},\left(x_{i_{1}}-x_{i_{2}}\right) x_{j},\left(x_{i_{1}}-x_{j}\right) x_{j}\right) \text {. }
$$


2) $\left(\kappa, \kappa^{\prime}\right)=\left((1,2,3),(i)\left[j_{1}, j_{2}\right]\right)$, where $\left\{i, j_{1}, j_{2}\right\}=\{1,2,3\}$ and $F=\tilde{F}\left(\left(x_{i}-x_{j_{1}}\right) x_{j_{1}},\left(x_{i}-x_{j_{1}}\right) x_{j_{2}},\left(x_{i}-x_{j_{2}}\right) x_{j_{1}},\left(x_{i}-x_{j_{2}}\right) x_{j_{2}},\left(x_{j_{1}}-x_{j_{2}}\right),\left(x_{j_{1}}-x_{j_{2}}\right) x_{i}\right)$.

3) $\left(\kappa, \kappa^{\prime}\right)=((1,2)[3],(i)[3, j])$, where $\{i, j\}=\{1,2\}$, and

$$
F=\tilde{F}\left(x_{3}, x_{j} x_{3}, x_{i} x_{3},\left(x_{i}-x_{j}\right) x_{j}\right) .
$$

4) $\left(\kappa, \kappa^{\prime}\right)=((1,2)[3],(3)[1,2])$ and

$$
F=\tilde{F}\left(x_{1} x_{3}, x_{2} x_{3}, x_{1}-x_{2}, r_{1} x_{1} x_{3}+r_{2} x_{2} x_{3}+r_{3}\left(x_{1}-x_{2}\right) x_{1}+r_{4}\left(x_{1}-x_{2}\right) x_{2}\right),
$$

where $r_{l} \in \mathbb{C}$, for $l=1, \cdots, 4$, such that $\left(r_{1} \neq 0, r_{2} \neq 0,\left(r_{3}, r_{4}\right) \neq(0,0)\right)$, or $\left(\left(r_{1}, r_{2}\right) \neq(0,0),\left(r_{3}, r_{4}\right) \neq(0,0)\right)$.

5) $\left(\kappa, \kappa^{\prime}\right)=((1,3)[2],(i)[2, j])$, where $\{i, j\}=\{1,3\}$, and

$$
F=\tilde{F}\left(x_{2}, x_{2} x_{j}, x_{i} x_{2},\left(x_{i}-x_{j}\right) x_{j}\right) .
$$

6) $\left(\kappa, \kappa^{\prime}\right)=((1,3)[2],(2)[1,3])$ and

$$
F=\tilde{F}\left(x_{1} x_{2}, x_{2} x_{3}, x_{1}-x_{3}, r_{1} x_{1} x_{2}+r_{2} x_{2} x_{3}+r_{3}\left(x_{1}-x_{3}\right) x_{1}+r_{4}\left(x_{1}-x_{3}\right) x_{3}\right),
$$

where $r_{l} \in \mathbb{C}$, for $l=1, \cdots, 4$, such that $\left(r_{1} \neq 0, r_{2} \neq 0\right)$, or $\left(r_{1}, r_{2}\right) \neq(0,0)$ and $\left(r_{3}, r_{4}\right) \neq(0,0)$.

7) $\left(\kappa, \kappa^{\prime}\right)=((2,3)[1],(i)[1, j])$, where $\{i, j\}=\{2,3\}$, and

$$
F=\tilde{F}\left(x_{1}, x_{1} x_{i}, x_{1} x_{j},\left(x_{i}-x_{j}\right) x_{j}\right) .
$$

8) $\left(\kappa, \kappa^{\prime}\right)=((2,3)[1],(1)[2,3])$ and

$$
F=\tilde{F}\left(x_{1} x_{3}, x_{1} x_{2}, x_{3}-x_{2}, r_{1} x_{1} x_{3}+r_{2} x_{1} x_{2}+r_{3}\left(x_{3}-x_{2}\right) x_{3}+r_{4}\left(x_{3}-x_{2}\right) x_{2}\right),
$$

where $r_{l} \in \mathbb{C}$, for $l=1, \cdots, 4$, such that $\left(r_{1} \neq 0, r_{2} \neq 0,\left(r_{3}, r_{4}\right) \neq(0,0)\right)$ or $\left(\left(r_{1}, r_{2}\right) \neq(0,0),\left(r_{3}, r_{4}\right) \neq(0,0)\right)$.

9) $\left(\kappa, \kappa^{\prime}\right)=((1)[2,3],(i)[1, j])$, where $\{i, j\}=\{2,3\}$, and

$$
F=\tilde{F}\left(x_{j}, x_{1} x_{i}, r_{1} x_{i} x_{j}+r_{2} x_{1} x_{j}\right),
$$

where $r_{1}$ et $r_{2}$ are the non-zero complex numbers.

B) We prove now that $S$ admits at most two façons. We prove the result for the first case of the above possibilities: $\left(\kappa, \kappa^{\prime}\right)=\left((1,2,3),\left(i_{1}, i_{2}\right)[j]\right)$, where $\left\{i_{1}, i_{2}, j\right\}=\{1,2,3\}$. The other cases are proved similarly. For example, assume that $S$ admits two façons $\kappa=(1,2,3)$ and $\kappa^{\prime}=(1,2)[3]$. We prove that $S$ cannot admit the third façon $\kappa^{\prime \prime}$ different from $\kappa$ and $\kappa^{\prime}$.

Let $\kappa^{\prime \prime}$ be a façon of $S_{F}$. Let us denote by $\left\{\xi_{k}^{\prime \prime}=\left(x_{1, k}^{\prime \prime}, x_{2, k}^{\prime \prime}, x_{3, k}^{\prime \prime}\right)\right\}$ a generic sequence corresponding to $\kappa^{\prime \prime}$. By the example 3.5, the mapping $F$ admits $X_{1}$, $X_{2}$ (or $X_{3}$ ), and $X_{5}$ as the pertinent variables, where

$$
X_{1}=x_{1}-x_{2}, X_{2}=\left(x_{1}-x_{2}\right) x_{1}, X_{3}=\left(x_{1}-x_{2}\right) x_{2}, X_{5}=\left(x_{1}-x_{3}\right) x_{3} \text {. }
$$

Without loose the generality, we can assume that $X_{2}$ is a pertinent variable of $F$. We prove that $X_{1}\left(\xi_{k}^{\prime \prime}\right)=\left(x_{1, k}^{\prime \prime}-x_{2, k}^{\prime \prime}\right)$ tends to 0 . Assume that $X_{1}\left(\xi_{k}^{\prime \prime}\right)=$ $\left(x_{1, k}^{\prime \prime}-x_{2, k}^{\prime \prime}\right)$ tends to a non-zero complex number. Then:

+ If $x_{1, k}^{\prime \prime}$ tends to infinity, then $X_{2}\left(\xi_{k}^{\prime \prime}\right)$ tends to infinity, that provides a contradiction with the fact that $X_{2}$ is a pertinent variable of $F$. 
+ If $x_{2, k}^{\prime \prime}$ tends to infinity, then $x_{1, k}^{\prime \prime}$ also tends to infinity since $\left(x_{1, k}^{\prime \prime}-x_{2, k}^{\prime \prime}\right)$ tends to a non-zero complex number. That implies $X_{2}\left(\xi_{k}^{\prime \prime}\right)$ tends to infinity and this provides a contradiction with the fact that $X_{2}$ is a pertinent variable of F.

Hence, $x_{1, k}^{\prime \prime}$ and $x_{2, k}^{\prime \prime} \quad$ cannot tend to infinity. Consequently, $x_{3, k}^{\prime \prime}$ must tend to infinity. Therefore, $X_{5}\left(\xi_{k}^{\prime \prime}\right)=\left(x_{1, k}^{\prime \prime}-x_{3, k}^{\prime \prime}\right) x_{3, k}^{\prime \prime}$ tends to infinity, that provides the contradiction with the fact that $X_{5}$ is a pertinent variable of $F$. We conclude that $\left(x_{1, k}^{\prime \prime}-x_{2, k}^{\prime \prime}\right)$ tends to 0 .

Then we have two possibilities:

a) either both of $x_{1, k}^{\prime \prime}$ and $x_{2, k}^{\prime \prime}$ tend to 0 : then $X_{1}\left(\xi_{k}^{\prime \prime}\right)=\left(x_{1, k}^{\prime \prime}-x_{2, k}^{\prime \prime}\right)$, $X_{2}\left(\xi_{k}^{\prime \prime}\right)=\left(x_{1, k}^{\prime \prime}-x_{2, k}^{\prime \prime}\right) x_{1, k}^{\prime \prime}$ and $X_{3}\left(\xi_{k}^{\prime \prime}\right)=\left(x_{1, k}^{\prime \prime}-x_{2, k}^{\prime \prime}\right) x_{2, k}^{\prime \prime}$ tend to 0 , which provides the contradiction with the fact that the dimension of $S$ is 2 ,

b) or both of $x_{1, k}^{\prime \prime}$ and $x_{2, k}^{\prime \prime}$ tend to infinity: Since $X_{5}$ is a pertinent variable of $F$, then $x_{3, k}^{\prime \prime}$ tends to 0 or infinity. We conclude that the façon $\kappa^{\prime \prime}$ is $(1,2,3)$ or $(1,2)[3]$.

In conclusion, $S$ admits only the two façons $\kappa=(1,2,3)$ and $\kappa^{\prime}=(1,2)[3]$.

c) We prove now that if there exists a 2-dimensional irreductible stratum $S$ of $S_{F}$ admitting two façons, then $S_{F}$ is a plane. Similarly to B), we prove this fact for the first case of the possibilities in A), that means, the case of $\left(\kappa, \kappa^{\prime}\right)=\left((1,2,3),\left(i_{1}, i_{2}\right)[j]\right)$, where $\left\{i_{1}, i_{2}, j\right\}=\{1,2,3\}$. The other cases are proved similarly. For example, assume that $S$ admits two façons $\kappa=(1,2,3)$ and $\kappa^{\prime}=(1,2)[3]$. With the same arguments than in the example 3.5, the stratum $S$ is a plane. By B), the asymptotic set $S_{F}$ admits also only two façons $\kappa=(1,2,3)$ and $\kappa^{\prime}=(1,2)[3]$. In other words, $S_{F}$ and $S$ concide. We conclude that $S_{F}$ is a plane.

We prove now the theorem 4.1.

Proof. (The proof of theorem 4.1). The cases 1) and 2) are easily achievable by the lemmas 4.4 and 4.2 , respectively. Let us prove the cases 3), 4) and 5). In these cases, on the one hand, since $S_{F}$ contains at least two irreductible surfaces, then $S_{F}$ admits at least two façons; on the other hand, by the lemma 4.4, each irreductible surface of $S_{F}$ admits only one façon. Assume that $\kappa, \kappa^{\prime}$ are two different façons of $S_{F}$ and $\left\{\xi_{k}\right\},\left\{\xi_{k}^{\prime}\right\}$ are two corresponding generic sequences, respectively. We use the algorithm 3.10 and in the same way than the proofs of the lemmas 4.2 and 4.4 , we can see easily that the pairs of façons $\left(\kappa, \kappa^{\prime}\right)$ must belong to only the following pairs of groups: (I, IV), (I, V), (I, VI), (II, VI), (IV, V), (IV, VI), (V, VI) and (VI, VI) in the list (3.4).

i) If $\kappa$ belongs to the group I and $\kappa^{\prime}$ belongs to the group IV, for example $\kappa=(1,2,3)$ and $\kappa^{\prime}=(1,2)[3]$. From the example $3.5, F$ is a dominant polynomial mapping which can be written in terms of pertinent variables:

$$
\begin{aligned}
& F=\tilde{F}\left(x_{1}-x_{2},\left(x_{1}-x_{2}\right) x_{1},\left(x_{1}-x_{2}\right) x_{2},\left(x_{1}-x_{2}\right) x_{3},\left(x_{1}-x_{3}\right) x_{3}\right) \\
& \lim _{k \rightarrow \infty} F\left(\xi_{k}\right)=\tilde{F}(0, \lambda, \lambda, \lambda, \mu), \\
& \lim _{k \rightarrow \infty} F\left(\xi_{k}^{\prime}\right)=\tilde{F}\left(0, \lambda^{\prime}, \lambda^{\prime}, 0, \mu^{\prime}\right),
\end{aligned}
$$

where $\lambda, \mu, \lambda^{\prime}, \mu^{\prime} \in \mathbb{C}$ (see (3.6), (3.7) and (3.8)). We see that, with the sequence 
$\left\{\xi_{k}\right\}$, the pertinent variables tending to an arbitrary complex numbers have the degree 2, then the façon $\kappa$ provides a plane, since the degree of $F$ is 2 . In the same way, the façon $\kappa^{\prime}$ provides a plane. Furthermore, it is easy to check that these two planes must have the form of the case 3) of the theorem and $S_{F}$ is the union of these two planes.

ii) If $\kappa$ belongs to the group I and $\kappa^{\prime}$ belongs to the group $V$, for example $\kappa=(1,2,3)$ and $\kappa^{\prime}=(1)[2]$. Then, on the one hand, $F$ is a dominant polynomial mapping which can be written in terms of pertinent variables:

$$
F=\tilde{F}\left(x_{2}-x_{3},\left(x_{2}-x_{3}\right) x_{2},\left(x_{2}-x_{3}\right) x_{3},\left(x_{1}-x_{2}\right) x_{2}\right) .
$$

On the other hand, with the same arguments than the example 3.5, and for suitable generic sequences $\left\{\xi_{k}\right\}$ and $\left\{\xi_{k}^{\prime}\right\}$, we obtain:

$$
\begin{aligned}
& \lim _{k \rightarrow \infty} F\left(\xi_{k}\right)=\tilde{F}(0, \lambda, \lambda, \mu), \\
& \lim _{k \rightarrow \infty} F\left(\xi_{k}^{\prime}\right)=\tilde{F}\left(\lambda^{\prime}, 0, \lambda^{\prime 2}, \mu^{\prime}\right),
\end{aligned}
$$

where $\lambda, \mu, \lambda^{\prime}, \mu^{\prime} \in \mathbb{C}$. With the same arguments than in the case i), we have:

a) either the façons $\kappa$ and $\kappa^{\prime}$ provide two planes of the form of the case 3) of our theorem,

b) or the façon $\kappa$ provides the plane $(\mathscr{T})$ and, by the lemma 4.2, the façon $\kappa^{\prime}$ provides the paraboloid ( $\left.\mathscr{C}\right)$ of the form of the case 4) of our theorem.

By an easy calculation, we see that if $S_{F}$ admits another façon $\kappa^{\prime \prime}$ which is different from the façons $\kappa$ and $\kappa^{\prime}$, then this façon provides a 1-dimensional stratum contained in $(\mathscr{P})$ or contained in $(\mathscr{C})$.

iii) Proceeding in the same way for the cases where $\left(\kappa, \kappa^{\prime}\right)$ is a pair of façons belonging to the pairs of groups: (I, VI), (II, VI), (IV, V), (IV, VI) and (V, VI), we obtain the case 3 ) or the case 4 ) of the theorem.

iv) Consider now the case where $\kappa$ and $\kappa^{\prime}$ belong to the group VI, for example, $\kappa=(1)[2,3]$ and $\kappa^{\prime}=(2)[1,3]$, then $F$ is a dominant polynomial mapping which can be written in terms of pertinent variables:

$$
F=\tilde{F}\left(x_{3}, x_{1} x_{2}, x_{2} x_{3}, x_{3} x_{1}\right) .
$$

With the same arguments than the example 3.5, and for suitable generic sequences $\left\{\xi_{k}\right\}$ and $\left\{\xi_{k}^{\prime}\right\}$, we obtain:

$$
\begin{aligned}
& \lim _{k \rightarrow \infty} F\left(\xi_{k}\right)=\tilde{F}(0, \lambda, 0, \mu), \\
& \lim _{k \rightarrow \infty} F\left(\xi_{k}^{\prime}\right)=\tilde{F}\left(0, \lambda^{\prime}, \mu^{\prime}, 0\right),
\end{aligned}
$$

where $\lambda, \mu, \lambda^{\prime}, \mu^{\prime} \in \mathbb{C}$. In this case, we have two possibilities:

a) either $F$ admits $x_{3}$ as a pertinent variable: This case is similar to the case i) and we have the case 3 ) of the theorem,

b) or $F$ does not admit $x_{3}$ as a pertinent variable, that means

$$
F=\tilde{F}\left(x_{1} x_{2}, x_{2} x_{3}, x_{3} x_{1}\right) \text {. }
$$

In this case, $S_{F}$ admits one more façon $\kappa^{\prime \prime}=(3)[1,2]$ such that with a corresponding suitable generic sequence $\left\{\xi_{k}^{\prime \prime}\right\}$ of $\kappa^{\prime \prime}$, we have 


$$
\begin{aligned}
& \lim _{k \rightarrow \infty} F\left(\xi_{k}\right)=\tilde{F}(\lambda, 0, \mu), \\
& \lim _{k \rightarrow \infty} F\left(\xi_{k}^{\prime}\right)=\tilde{F}\left(\lambda^{\prime}, \mu^{\prime}, 0\right), \\
& \lim _{k \rightarrow \infty} F\left(\xi_{k}^{\prime \prime}\right)=\tilde{F}\left(0, \lambda^{\prime \prime}, \mu^{\prime \prime}\right),
\end{aligned}
$$

where $\lambda^{\prime \prime}, \mu^{\prime \prime} \in \mathbb{C}$. In this case, $S_{F}$ is the union of three planes the forms of which are as in the case 5) of the theorem.

\section{The General Case}

The algorithm 3.10 can be generalized to clasify the asymptotic sets of nonproper dominant polynomial mappings $F: \mathbb{C}^{n} \rightarrow \mathbb{C}^{n}$ of degree $d$ where $n \geq 3$ and $d \geq 2$ as the following.

Algorithm 5.1. We have the six following steps:

Step 1: Determine the list $\mathcal{L}_{F}^{(n, d)}$ of all the possible façons of $S_{F}$.

Step 2: Fix $I$ façons $\kappa_{1}, \cdots, \kappa_{l}$ in the list $\mathcal{L}_{F}^{(n, d)}$ obtained in step 1. Determine the pertinent variables with respect to these $I$ façons (in the similar way than the definition 3.1).

Step 3:

- Assume that $S$ is a $(n-1)$-dimensional stratum of $S_{F}$ admiting only the $I$ façons $\kappa_{1}, \cdots, \kappa_{l}$ determined in step 1 .

- Take generic sequences $\xi_{k}^{1}, \cdots, \xi_{k}^{l}$ corresponding to $\kappa_{1}, \cdots, \kappa_{l}$, respectively.

- Compute the limit of the images of the sequences $\xi_{k}^{1}, \cdots, \xi_{k}^{l}$ by pertinent variables defined in step 1.

- Restrict the pertinent variables defined in step 2 using the fact $\operatorname{dim} S=n-1$.

Step 4: Restrict again the pertinent variables in step 3 using the three following facts:

- all the façons $\kappa_{1}, \cdots, \kappa_{l}$ belong to $S$ : then the images of the generic sequences $\xi_{k}^{1}, \cdots, \xi_{k}^{l}$ by the pertinent variables defined in the step 2 must tend to either an arbitrary complex number or zero,

- $\operatorname{dim} S=n-1:$ then there are at least $n-1$ pertinent variables $X_{i_{1}}, \cdots, X_{i_{n-1}}$ such that the images of the sequences $\left\{\xi_{k}^{1}\right\}, \cdots,\left\{\xi_{k}^{l}\right\}$ by $X_{i_{1}}, \cdots, X_{i_{n-1}}$, respectively, tend independently to $(n-1)$ complex numbers,

- $F$ is dominant: then there are at least $n$ independent pertinent variables (see lemma 2.2).

Step 5: Describe the geometry of the $(n-1)$-dimensional irreductible stratum $S$ in terms of the pertinent variables obtained in the step 4 .

Step 6: Let $I$ run in the list obtained in the step 1.

Theorem 5.2. With the algorithm 5.1, we obtain all possible asymptotic sets of non-proper dominant polynomial mapping $F: \mathbb{C}^{n} \rightarrow \mathbb{C}^{n}$ of degree $d$.

Proof. In the one hand, by theorem 2.4 , the dimension of $S_{F}$ is $n-1$. By the step 3, step 5 and step 6 , we consider all the possible cases of the all $(n-1)$ dimensional irreductible strata of $S_{F}$. Since the dimension of $S_{F}$ is $n-1$ (see theorem 2.4), we get all the possible asymptotic sets $S_{F}$ of non-proper dominant polynomial mappings $F: \mathbb{C}^{n} \rightarrow \mathbb{C}^{n}$ of degree $d$. In the other hand, the number of all the possible façons of a polynomial mapping 
$F: \mathbb{C}^{n} \rightarrow \mathbb{C}^{n}$ is finite, as the shown of the following lemma:

Lemma 5.3. Let $F: \mathbb{C}^{n} \rightarrow \mathbb{C}^{n}$ be a polynomial mapping such that $S_{F} \neq \varnothing$. Then, the number of all possible façons of $S_{F}$ is finite. More precisely, the maximum number of façons of $S_{F}$ is equal to

$$
\sum_{t=1}^{n} C_{t}^{n}+\sum_{t=1}^{n-1} C_{t}^{n}+\sum_{t=2}^{n-1} A_{t}^{n}
$$

where

$$
C_{t}^{n}=\frac{n !}{t !(n-t) !}, \quad A_{t}^{n}=\frac{n !}{(n-t) !} .
$$

Proof. Assume that $\kappa=\left(i_{1}, \cdots, i_{p}\right)\left[j_{1}, \cdots, j_{q}\right]$ is a façon of $S_{F}$. We have the following cases:

i) If $\left\{i_{1}, \cdots, i_{p}\right\} \cup\left\{j_{1}, \cdots, j_{q}\right\}=\{1, \cdots, n\}$ : we have $\sum_{t=1}^{n} C_{t}^{n}$ possible façons.

ii) If $\left\{i_{1}, \cdots, i_{p}\right\} \cup\left\{j_{1}, \cdots, j_{q}\right\} \neq\{1, \cdots, n\}$ and $\left\{j_{1}, \cdots, j_{q}\right\}=\varnothing$ : we have $\sum_{t=1}^{n-1} C_{t}^{n}$ possible façons.

iii) If $\left\{i_{1}, \cdots, i_{p}\right\} \cup\left\{j_{1}, \cdots, j_{q}\right\} \neq\{1, \cdots, n\}$ and $\left\{j_{1}, \cdots, j_{q}\right\} \neq \varnothing$ : We have $\sum_{t=2}^{n-1} A_{t}^{n}$ possible façons.

As the three cases are independent, then the maximum number of façons of $S_{F}$ is equal to

$$
\sum_{t=1}^{n} C_{t}^{n}+\sum_{t=1}^{n-1} C_{t}^{n}+\sum_{t=2}^{n-1} A_{t}^{n}
$$

Remark 5.4. In the example 3.5 and in the proofs of the lemmas 4.2 and 4.4, we use a linear change of variables to simplify the pertinent variables (so that we can work without coefficients and then we can simplify calculations). This change of variables does not modify the results of the theorem 4.1. However, in the algorithms 3.10 and 5.1, we do not need the step of linear change of variables, since the computers can work with coefficients of pertinent variables without making the problem heavier.

\section{References}

[1] Jelonek, Z. (1993) The Set of Points at Which Polynomial Map Is Not Proper. Annales Polonici Mathematici, 58, 259-266.

[2] Nguyen, T B.T. (2016) La méthode des façons. https://arxiv.org/pdf/1407.5329.pdf 
Submit or recommend next manuscript to SCIRP and we will provide best service for you:

Accepting pre-submission inquiries through Email, Facebook, LinkedIn, Twitter, etc. A wide selection of journals (inclusive of 9 subjects, more than 200 journals)

Providing 24-hour high-quality service

User-friendly online submission system

Fair and swift peer-review system

Efficient typesetting and proofreading procedure

Display of the result of downloads and visits, as well as the number of cited articles Maximum dissemination of your research work

Submit your manuscript at: http://papersubmission.scirp.org/

Or contact ajcm@scirp.org 